\title{
Effects of kartogenin on the attenuated nucleus pulposus cell degeneration of intervertebral discs induced by interleukin-1 $\beta$ and tumor necrosis factor- $\alpha$
}

\author{
YAO HUANG, TAO JIANG, JIAN CHEN, GUO-YONG YIN and JIN FAN \\ Department of Orthopaedics, The First Affiliated Hospital of Nanjing Medical University, \\ Nanjing, Jiangsu 210000, P.R. China
}

Received June 17, 2016; Accepted November 15, 2017

DOI: $10.3892 /$ ijmm.2017.3283

\begin{abstract}
Cytokines are the main cause of intervertebral disc degeneration. Kartogenin (KGN) is found to protect chondrocytes from cytokines. To explore whether KGN can slow down the degeneration on intervertebral discs following exposure to interleukin-1 $\beta$ (IL-1 $\beta$ ) and tumor necrosis factor- $\alpha$ (TNF- $\alpha$ ), the expression of type II collagen (Col II) and aggrecan were detected by immunofluorescence, immunohistochemistry and tissue staining. An in vitro model of disc degeneration using human nucleus pulposus cells (hNPCs) and ex vivo culture of mouse intervertebral discs organs under the actions of inflammatory cytokines were used, and the expression of Col II and aggrecan in hNPCs were detected by semi-quantitative western blot analysis, and the mRNA expression of the genes than encode Col II and aggrecan were detected by reverse transcription-quantitative polymerase chain reaction (RT-qPCR). The results indicated that the expression of $\mathrm{Col}$ II and aggrecan was reduced in the degeneration models. However, the protein expressions of Col II and aggrecan were significantly elevated in hNPCs and the mouse intervertebral discs following addition of KGN. RT-qPCR results revealed that the mRNA expression of $\mathrm{Col}$ II and aggrecan was increased in hNPCs and mouse intervertebral discs following treatment with KGN. Thus, KGN effectively increased the expression of Col II and aggrecan in hNPCs and slowed the degeneration of intervertebral discs stimulated by IL- $1 \beta$ and TNF- $\alpha$.
\end{abstract}

\section{Introduction}

Lower back pain affects $70-85 \%$ of the American population daily (1), resulting in annual government spending of

Correspondence to: Professor Guo-Yong Yin or Professor Jin Fan, Department of Orthopaedics, The First Affiliated Hospital of Nanjing Medical University, 300 Guangzhou Road, Nanjing, Jiangsu 210000, P.R. China

E-mail: guoyong_yin@sina.com

E-mail: fanjin@njmu.edu.cn

Key words: kartogenin, interleukin-1 $\beta$, tumor necrosis factor- $\alpha$, slow down, intervertebral disc, degeneration
$>\$ 100$ billion on health care (2). This pain may have discogenic, neurogenic and ormyogenic causes, among others (3). The degeneration of intervertebral discs is a major cause of lower back pain as a result of the upregulated expression of inflammatory cytokines (4-7). Therefore, inflammatory cytokines have received increasing attention from researchers; specifically, interleukin-1 $\beta$ (IL-1 $\beta$ ) and tumor necrosis factor- $\alpha(\mathrm{TNF}-\alpha)$ are used to establish models of intervertebral disc degeneration (8).

The degeneration of intervertebral discs is often accompanied by the downregulated expression of type II collagen (Col II) and aggrecan (9). Col II and aggrecan are predominantly secreted by nucleus pulposus cells (NPCs), and are used as phenotypic markers for NPCs. The downregulated expression of $\mathrm{Col}$ II and aggrecan reduces the elastic modulus of intervertebral discs (10). Water loss and height reduction cause the degeneration of intervertebral discs to occur $(10,11)$.

Several studies have discussed the treatment of intervertebral disc degeneration (12); these approaches include stem cell transplantation, growth hormone injection and physiotherapy (13). However, only a few of these methods are widely accepted given the presence of wounds and poor biosafety (14). No safe and effective drug has been identified as an effective treatment for intervertebral disc degeneration. Kartogenin (KGN) is a recently discovered low-molecular weight molecule that induces the differentiation of bone marrow mesenchymal stem cells (MSCs) into chondrocytes via the core binding factor $\beta(\mathrm{CBF} \beta)$-runt related transcription factor 1 (RUNX1) pathway (15). KGN also promotes the expression of Col II and aggrecan in chondrocytes (16). KGN may be useful for treating osteoarthritis. Recently, KGN was reported to effectively repair articular cartilage damage (17). However, its use for the treatment of the degeneration of intervertebral discs has not been reported. In the present study, KGN was used to address the difficulty of treating degeneration in the intervertebral discs.

\section{Materials and methods}

Experimental subjects. Human NPCs (hNPCs) were purchased from ScienCell Research Laboratories, Inc. (San Diego, CA, 
USA; cat. no. 4800). Laboratory animals used in the present study were 6-week-old male B6 mice provided by the Model Animal Research Center of Nanjing University (Nanjing, China), which has a certificate of conformity (201500796). The present study followed the Ethics Codes for Laboratory Animals was approved by the Ethics Committee of the First Affiliated Hospital of Nanjing Medical University (Nanjing, China).

Culture of hNPCs and the organ culture of mouse intervertebral discs. The hNPCs were passaged when $80 \%$ of the dish was covered. The first three generations of these cells were used for the experiments. For the organ culture of mouse intervertebral discs, mice under carbon dioxide anesthesia were sacrificed by cervical dislocation, and soaked in $75 \%$ alcohol for $5 \mathrm{~min}$ before sterile operation on a clean bench. Segments were removed from the lumbar spines of the mice with back-cuts. The intervertebral discs L2-3 and L3-4 (including the upper and lower endplates) were dissected and cultured on a 6 -well plate, with $4 \mathrm{ml}$ culture medium added to each plate. The hNPCs and mouse intervertebral discs were cultured in an incubator containing $5 \% \mathrm{CO}_{2}$ and at $37^{\circ} \mathrm{C}$, with the medium changed every other day. The culture media for the hNPCs is the special media purchased from ScienCell Research Laboratories (cat. no. 4801). The culture media for the mouse intervertebral discs contained $10 \%$ fetal bovine serum (Hyclone; GE Healthcare Life Sciences, Logan, UT, USA), $1 \%$ double-antibiotic (streptomycin + penicillin; Gibco; Thermo Fisher Scientific, Inc., Waltham, MA, USA), and 1:1 Dulbecco's modified Eagle's medium (DMEM)/F12 (Hyclone; GE Healthcare Life Sciences) in the volume fraction.

Treatment groups. The hNPCs were divided into the following treatments groups: Group 1, blank control; group 2, treatment with inflammatory cytokines $10 \mathrm{ng} / \mathrm{ml} \mathrm{IL-1 \beta}$ and $25 \mathrm{ng} / \mathrm{ml}$ TNF- $\alpha$ (both from PeproTech, Inc., Rocky Hill, NJ, USA); group 3, treated with $10 \mathrm{ng} / \mathrm{ml}$ IL-1 $\beta, 25 \mathrm{ng} / \mathrm{ml}$ TNF- $\alpha$ and $100 \mathrm{nmol} / \mathrm{ml} \mathrm{KGN}$ [dissolved in dimethyl sulfoxide (DMSO); Selleck Chemicals, Houston, TX, USA]; group 4, treated with $10 \mathrm{ng} / \mathrm{ml} \mathrm{IL-1} \beta, 25 \mathrm{ng} / \mathrm{ml} \mathrm{TNF}-\alpha$ and $1 \mu \mathrm{mol} / \mathrm{ml} \mathrm{KGN}$. All hNPCs were cultured for 24 and $48 \mathrm{~h}$. The mouse intervertebral disc organ cultures were grouped as follows: Group 1, blank control; group 2, treatment with inflammatory cytokines $10 \mathrm{ng} / \mathrm{ml} \mathrm{IL-1 \beta ,50} \mathrm{ng/ml} \mathrm{TNF-} \alpha$; group 3, treated with $10 \mathrm{ng} / \mathrm{ml} \mathrm{IL-1 \beta ,} 25 \mathrm{ng} / \mathrm{ml} \mathrm{TNF}-\alpha$ and $100 \mathrm{nmol} / \mathrm{ml} \mathrm{KGN}$; group 4 , treated with $10 \mathrm{ng} / \mathrm{ml} \mathrm{IL-1} \beta, 25 \mathrm{ng} / \mathrm{ml} \mathrm{TNF-} \alpha$ and $1 \mu \mathrm{mol} / \mathrm{ml} \mathrm{KGN}$. The mouse intervertebral discs were cultured for 3 and 10 days. Each group of hNPCs or mouse intervertebral discs received equal amounts of the culture medium with DMSO (Sigma-Aldrich; Merck KGaA, Darmstadt, Germany).

Immunofluorescence, histology and immunohistochemistry. For the immunofluorescence assays, hNPCs were cultured in a 24-well plate, rinsed three times with phosphate-buffered saline (PBS; $3 \mathrm{~min}$ for each rinse), and fixed for $30 \mathrm{~min}$ with $4 \%$ paraformaldehyde at room temperature (RT). The excess paraformaldehyde was discarded, cells were rinsed with PBS and incubated with $0.5 \%$ Triton X-100 (Sigma-Aldrich; Merck $\mathrm{KGaA}$ ) for $15 \mathrm{~min}$ at $\mathrm{RT}$ to permeablize the membranes. The cells were blocked with goat serum (Beyotime Institute of Biotechnology, Haimen, China) for $1 \mathrm{~h}$ at RT and rinsed with PBS before the primary antibodies [anti-Collagen III (ab34712) and anti-aggrecan (ab36861); diluted 1:100; Abcam, Cambridge, UK] was applied for incubation at $4^{\circ} \mathrm{C}$ overnight. Following rinsing with PBS, the secondary antibodies [Cy3-conjugated anti-rabbitIgG (A0516) and Alexa Fluor 488-labeled goat anti-rabbit IgG (A0423); diluted by 1:100; Beyotime Institute of Biotechnology] was applied and the cells were incubated for $1 \mathrm{~h}$ in the dark at RT. Finally, the cells were rinsed with PBS, and the nuclei were counterstained with DAPI for $15 \mathrm{~min}$ at RT. The stained cells were imaged using a fluorescence microscope.

For cell histology and immunohistochemistry, intervertebral discs and the two endplates were fixed with $10 \%$ paraformaldehyde for $24 \mathrm{~h}$ at RT, dehydrated with a graded ethanol series $(50 \%$ ethanol for $1 \mathrm{~h} ; 50 \%$ ethanol for $1 \mathrm{~h} ; 70 \%$ ethanol for $1 \mathrm{~h} ; 80 \%$ ethanol for $1 \mathrm{~h} ; 90 \%$ ethanol for $1 \mathrm{~h}$; $95 \%$ ethanol for $1 \mathrm{~h}$; No.1 anhydrous ethanol for $1 \mathrm{~h}$; No.2 anhydrous alcohol for $1 \mathrm{~h}$; anhydrous ethanol: xylene $1: 1$ for $1 \mathrm{~h}$ ), and embedded in paraffin after decalcification with $12.5 \%$ ethylenediaminetetraacetic acid ( $\mathrm{pH} 7.4)$ at RT for 28 days. The $5 \mu \mathrm{m}$-thick paraffin sections were dewaxed, dehydrated, and stained with Alcian blue at RT for $15 \mathrm{~min}$, as well as hematoxylin (at RT for $10 \mathrm{~min}$ ) and eosin (at RT for $1 \mathrm{~min})(\mathrm{H} \& \mathrm{E})$. In the results, the cells were considered positive when the intervertebral discs were stained blue, and negative when they were colorless.

For the Col II immunohistochemistry, the paraffin sections were de-waxed, dehydrated, and incubated overnight at $4^{\circ} \mathrm{C}$ with anti-Col II (ab34712; diluted by 1:200; Abcam). After the primary antibody was removed, the secondary antibody (Q-11401MP; diluted by 1:100; Thermo Fisher Scientific, Inc.) was added for $1 \mathrm{~h}$ at room temperature. The sections were washed three times with PBS, with 3 min for each rinse. The stained cells were developed with diaminobenzidine at RT for $5 \mathrm{~min}$, counterstained with hematoxylin at RT for $10 \mathrm{~min}$, and mounted with a conventional medium when they became transparent after dehydration. The results revealed that the brownish yellow cells were Col II-positive, whereas the colorless cells were negative.

$R N A$ extraction and reverse transcription-quantitative polymerase chain reaction ( $R T-q P C R)$. Total RNA was respectively extracted from hNPCs and the nucleus pulposus tissues of the intervertebral discs of 3- and 10-day-old cultures with an RNA extraction kit (Bioteke Corporation, Beijing, China). Following quantification, the RNA was reverse transcribed into first strand cDNA with an iScript cDNA kit (Bio-Rad Laboratories, Inc., Hercules, CA, USA) with a $10 \mu 1$ reaction volume. qPCR was determined using SYBR-Green PCR Master Mix (ABI, Foster City, CA, USA). The samples were treated with recombinant DNase I (DNA-free DNA removal kit; Ambion, Austin, TX, USA) to remove possible DNA contamination. $\beta$-actin was used as an internal control. The PCR cycling conditions were as follows: Initial pre-denaturation of $95^{\circ} \mathrm{C}$ for $1 \mathrm{~min}$, denaturation for $15 \mathrm{sec}$, annealing of $55-65^{\circ} \mathrm{C}$ for $20 \mathrm{sec}$ and extension of $72^{\circ} \mathrm{C}$ for $30 \mathrm{sec}$. A total of 50 cycles were performed. For RT-qPCR the following primers were used: $\beta$-actin promoter forward (human), 5'-CATGTACGTTGCTATCCAGGC-3' and reverse, 
A

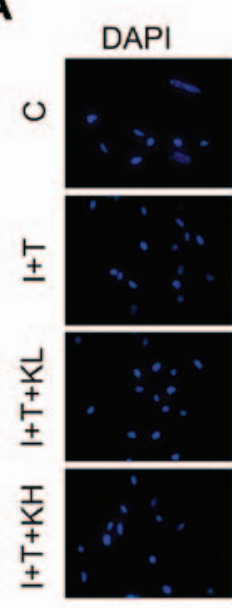

C
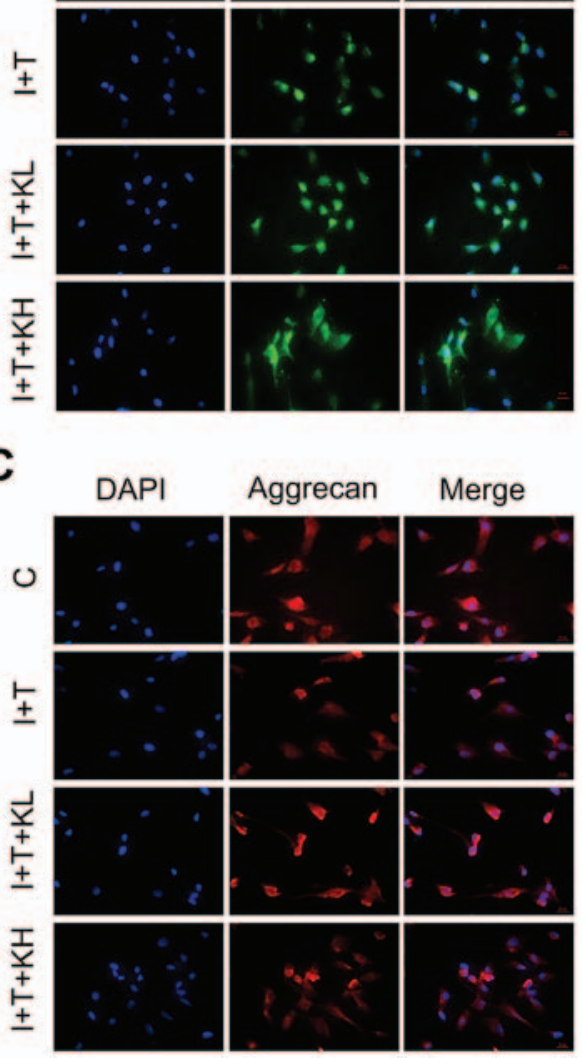

B

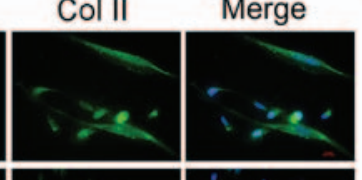

D

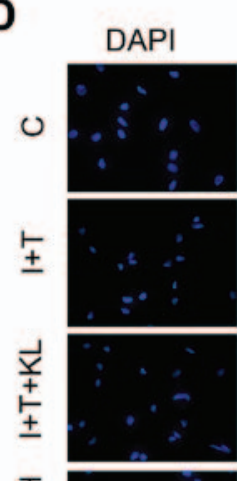

Aggrecan

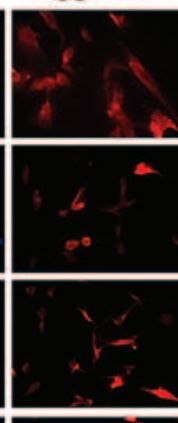

Merge

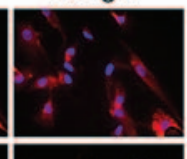

$\frac{I}{y}$
$\frac{5}{ \pm}$
\pm
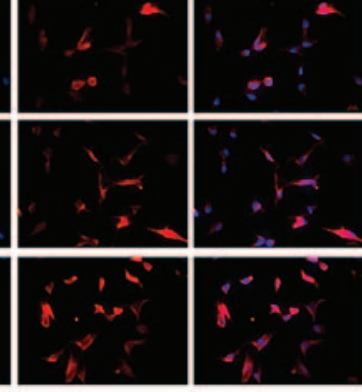

Figure 1. Col II and aggrecan expression were elevated by KGN in hNPCs. Expression of Col II and aggrecan in hNPCs after 24 and 48 h by immunofluorescence. (A) Col II, 24 h; (B) Col II, 48 h; (C) aggrecan, 24 h; and (D) aggrecan, 48 h. The expression of Col II and aggrecan were significantly inhibited in the group under inflammatory cytokines alone. However, the expression of Col II and aggrecan were elevated by KGN (original magnification, x200). Col II, type II collagen; KGN, kartogenin; hNPC, human nucleus pulposus cells; IL-1 $\beta$, interleukin-1 $\beta$; TNF- $\alpha$, tumor necrosis factor- $\alpha$; C, control; I+T, IL-1 $\beta+$ TNF- $\alpha$; $\mathrm{I}+\mathrm{T}+\mathrm{KL}, \mathrm{IL}-1 \beta+\mathrm{TNF}-\alpha+$ low concentration of KGN; I+T+KH, IL-1 $\beta+\mathrm{TNF}-\alpha+$ high concentration of KGN.

5'-CTCCTTAATGTCACGCACGAT-3'; Col2a1 promoter forward (human), 5'-TGGACGATCAGGCGAAACC-3' and reverse, 5'-GCTGCGGATGCTCTCAATCT-3'; aggrecan promoter forward (human), 5'-TCAACTGCTGCAGAC CAGGAGGT-3' and reverse, 5'-CCGATCCACTGGTAG TCTTGGGCA-3'; RUNX2 promoter forward (human), 5'-CCGCCTCAGTGATTTAGGGC-3' and reverse, 5'-GGG TCTGTAATCTGACTCTGTCC-3'; $\beta$-actinpromoter forward (mouse), 5'-AACAGTCCGCCTAGAAGCAC-3' and reverse, 5'-CGTTGACATCCGTAAAGACC-3'; Col2a1 promoter forward (mouse), 5'-CAGGATGCCCGAAAATTAGGG-3 and reverse, 5'-ACCACGATCACCTCTGGGT-3'; aggrecan promoter forward (mouse), 5'-GTGGAGCCGTGTTTCC AAG-3' and reverse, 5'-AGATGCTGTTGACTCGAACCT-3'; RUNX2 promoter forward (mouse), 5'-GACTGTGGTTAC CGTCATGGC-3' and reverse, 5'-ACTTGGTTTTTCATA ACAGCGGA-3'. Based on the $\mathrm{Cq}$ value and relative standard curve of the PCR product, the amount of RNA template contained in each specimen was determined and compared with the amount of $\beta$-actin. The specific value of the amount of RNA template to that of $\beta$-actin was adopted as the final statistical data. The results were processed using the $\Delta \Delta \mathrm{Cq}$ method (18).
Western blot analysis. The hNPCs after 24 and $48 \mathrm{~h}$, and NP cultures of mouse intervertebral discs after 3 and 10 days, for the different treatment groups, were lysed (RIPA; Beyotime Institute of Biotechnology) and centrifuged at $500 \mathrm{x} \mathrm{g}$ for 15 min. Following the quantification of the supernatant (BCA method), cell proteins (30 $\mu \mathrm{g}$ per lane) were isolated by 5 and $6 \%$ polyacrylamide gel electrophoresis at $110 \mathrm{~V}$ and electrophoretically transferred onto PVDF membranes. The membranes were blocked for $2 \mathrm{~h}$ with $5 \%$ bovine serum albumin reagent (Beyotime Institute of Biotechnology) and incubated overnight at $4^{\circ} \mathrm{C}$ with the primary antibodies (ab34712 and ab36861, diluted by 1:1,000; Abcam) before incubation for $120 \mathrm{~min}$ at room temperature with the secondary antibody (goat anti-rabbit antibody IgG; Q-11401MP; 1:5,000; Thermo Fisher Scientific, Inc.) and development for further analysis.

Statistical analysis. SPSS 19.0 statistical software (SPSS, Inc., Chicago, IL, USA) was used for all statistical analysis. The measurements were presented as mean \pm standard deviation, and the data between groups were analyzed using one-way analysis of variance (ANOVA) followed by a Bonferroni's posthoc test for multiple comparisons. $\mathrm{P}<0.05$ was considered to indicate a statistically significant difference. 
A

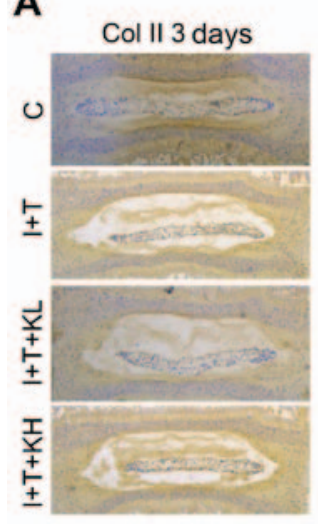

B

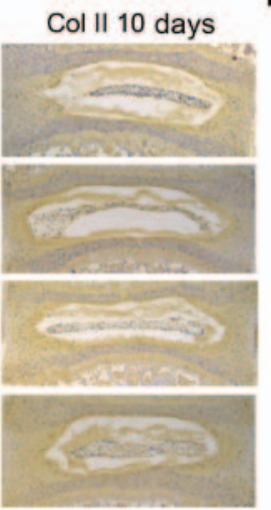

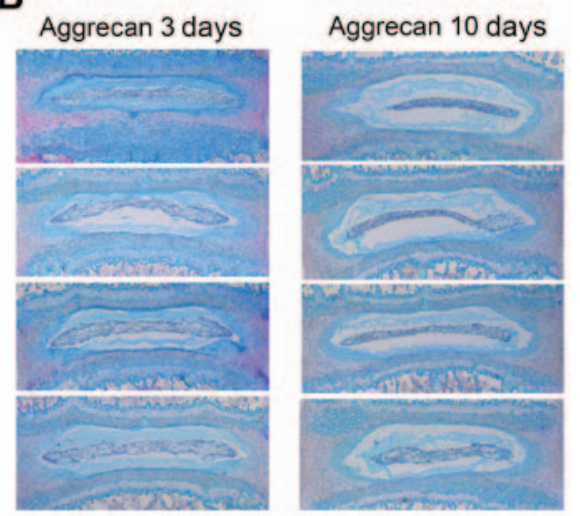

Figure 2. KGN promoted the expression of Col II and aggrecan in mouse intervertebral discs. (A) Immunohistochemisty of Col II and (B) aggrecan staining in paraffin sections of mouse intervertebral discs (at 3 and 10 days). Col II expression in the group treated with KGN was higher than that in the group under inflammatory cytokines. The group under inflammatory cytokines had a collapsed nucleus pulposus, thereby indicating the extracellular matrix loss in the intervertebral disc. However, the group with KGN did not exhibit such a phenomenon, with less loss of the extracellular matrix from the nucleus pulposus tissues in the intervertebral discs (original magnification, $\mathrm{x} 40$ ). Col II, type II collagen; KGN, kartogenin; IL-1 $\beta$, interleukin-1 $\beta$; TNF- $\alpha$, tumor necrosis factor- $\alpha$; C, control; I+T, IL-1 $\beta+$ TNF- $\alpha$; I+T+KL, IL-1 $\beta+$ TNF- $\alpha+$ low concentration of KGN; I+T+KH, IL-1 $\beta+$ TNF- $\alpha+$ high concentration of KGN.

A

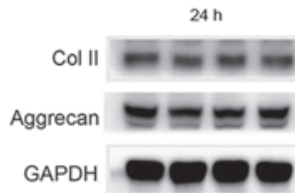

C

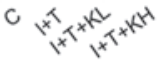
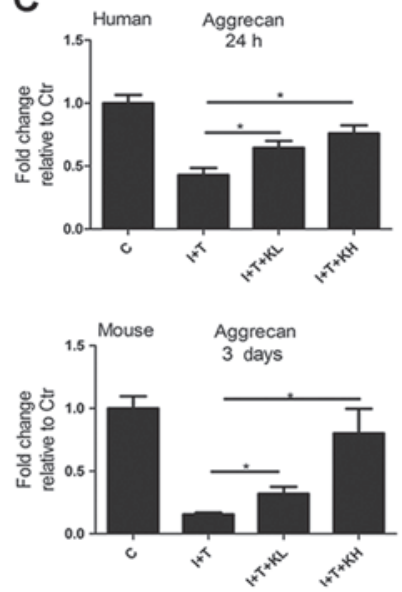

$48 \mathrm{~h}$
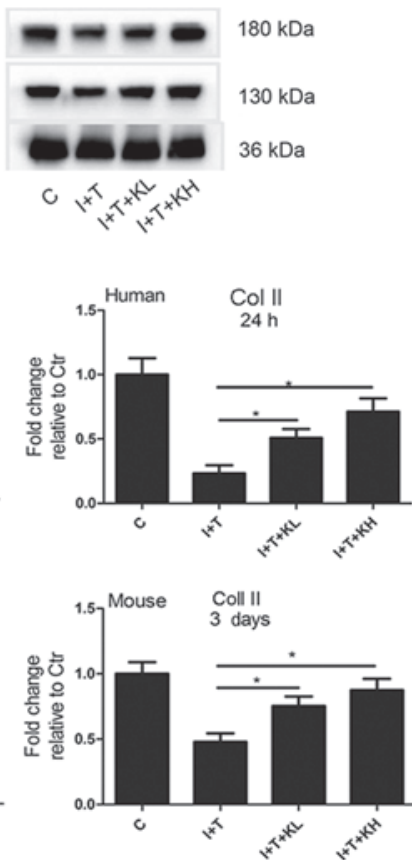

B
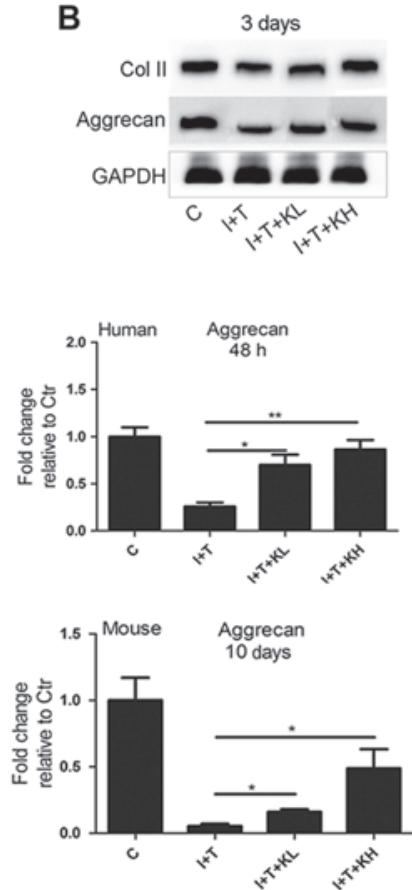

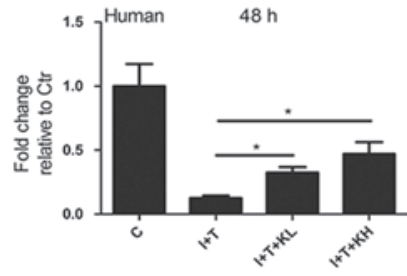

10 days
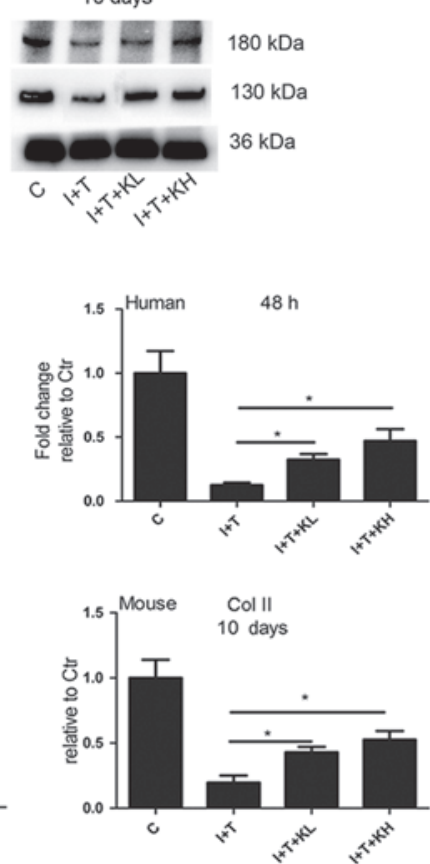

Figure 3. KGN promoted the release of Col II and aggrecan. (A) Western blot analysis of Col II and aggrecan expression in hNPCs (at 24 and 48 h). (B) Expression of Col II and aggrecan in the nucleus pulposus of mouse intervertebral discs by western blot analysis (at 3 and 10 days). The expression of Col II and aggrecan in the groups with KGN (especially at a high concentration) were higher than those in the group under inflammatory cytokines but did not reach the levels of the blank control group. (C) Densitometry of western blot analysis showed KGN improved the expression of Col II and aggrecan in hNPCs and intervertebral discs. Data presented as mean \pm standard deviation of three independent experiments performed in triplicate ( $\mathrm{n}=3$ ). ${ }^{*} \mathrm{P}<0.05$ and ${ }^{* *} \mathrm{P}<0.01$. Col II, type II collagen; KGN, kartogenin; hNPC, human nucleus pulposus cells; IL-1 $\beta$, interleukin-1 $\beta$; TNF- $\alpha$, tumor necrosis factor- $\alpha$; C, control; I+T, IL-1 $\beta+$ TNF- $\alpha$; I+T+KL, IL-1 $\beta+$ TNF- $\alpha+$ low concentration of KGN; I+T+KH, IL-1 $\beta+$ TNF- $\alpha+$ high concentration of KGN.

\section{Results}

Effects of KGN on Col II and aggrecan secretion. Immunohistochemistry and semi-quantitative western blot analysis were performed to observe variations of Col II in the intervertebral disc cultures. As shown by the immunofluorescence of Col II in hNPCs (Fig. 1A and B), the Col II expression was highest in the blank control group. However, the expression of Col II was inhibited by adding IL-1 $\beta$ and TNF- $\alpha$.
Col II expression was significantly elevated by the addition of KGN, particularly at a high concentration. To observe variations in the secretion of aggrecan by hNPCs, the expression of aggrecan in hNPCs was determined by immunofluorescence. As Fig. 1 shows, the aggrecan expression in the group under inflammatory cytokines was significantly inhibited within 24 and $48 \mathrm{~h}$ (Fig. 1C and D). However, by adding KGN, the expression was elevated, particularly in the group treated with a high concentration of $\mathrm{KGN}$. 
A
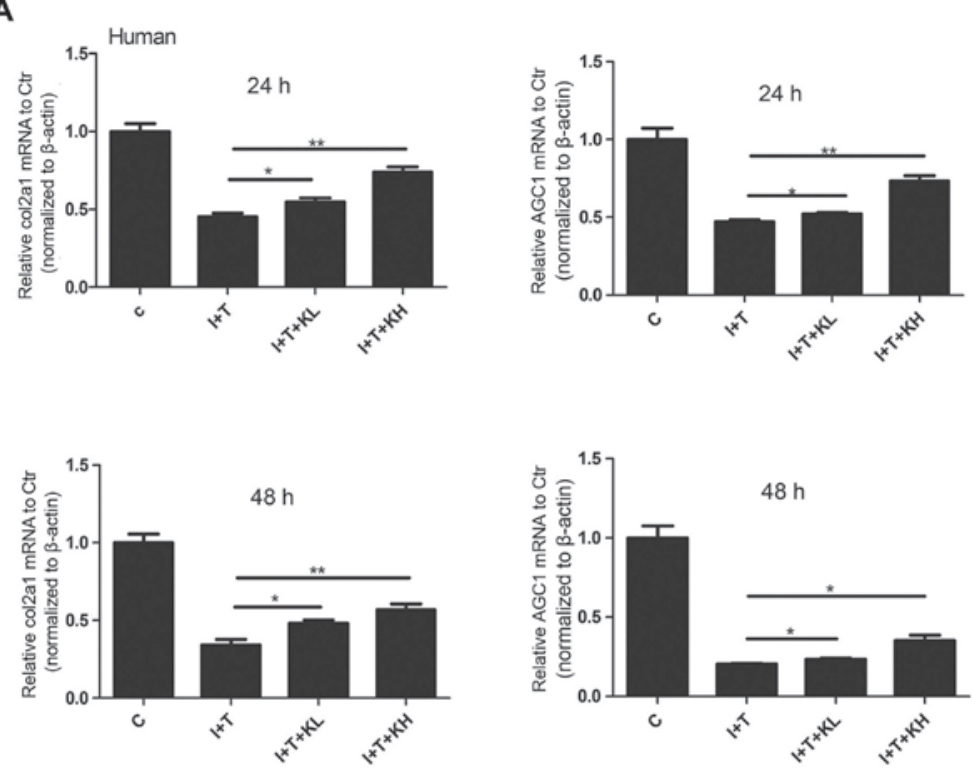

B
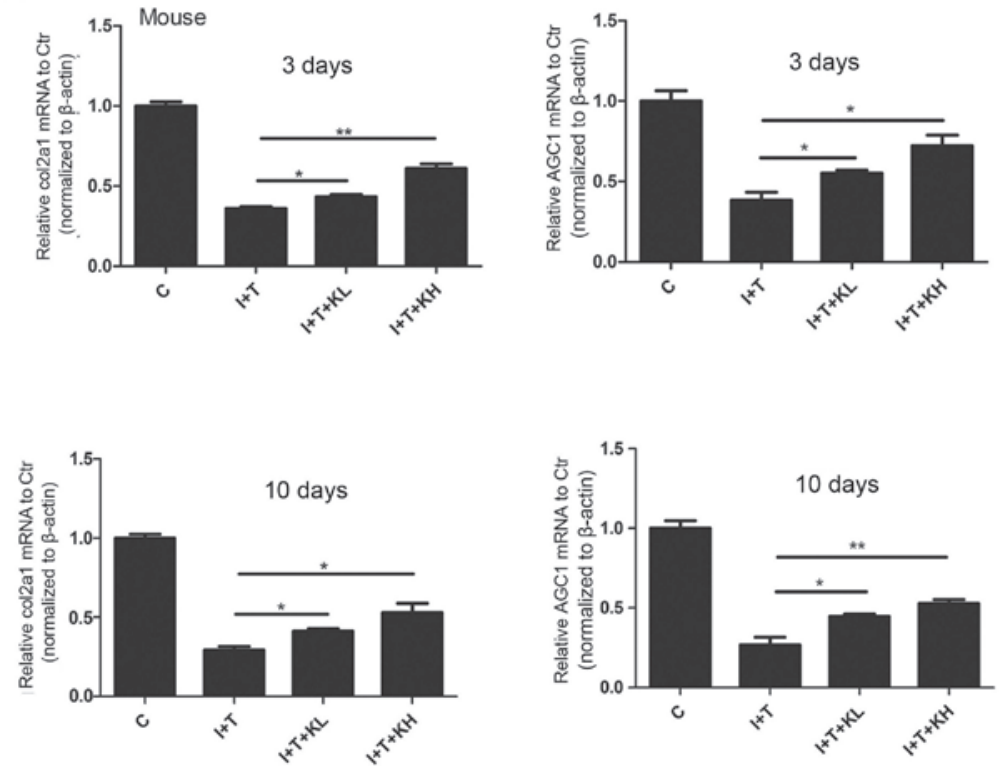

Figure 4. KGN increased the mRNA expression of Col II and aggrecan. Reverse transcription-quantitative polymerase chain reaction of genes associated with Col II and aggrecan in (A) human nucleus pulposus cells and (B) mouse intervertebral discs. The inflammatory cytokines significantly inhibited the expression of genes associated with Col II and aggrecan. Their expression was elevated by adding KGN; although their expression levels did not reach that of the blank control group, the difference was still statistically significant compared with the group under inflammatory cytokines ( ${ }^{*} \mathrm{P}<0.05$ and $\left.{ }^{* *} \mathrm{P}<0.001\right)$. Col II, type II collagen; col2a1, collagen type II $\alpha 1$ chain; AGC1, aggrecan; KGN, kartogenin; IL-1 $\beta$, interleukin-1 $\beta$; TNF- $\alpha$, tumor necrosis factor- $\alpha$; C, control; I+T, IL-1 $\beta+$ TNF- $\alpha$; I+T+KL, IL-1 $\beta+$ TNF- $\alpha+$ low concentration of KGN; I+T+KH, IL-1 $\beta$ + TNF- $\alpha$ + high concentration of KGN.

Similarly, from the immunohistochemistry analysis of paraffin sections of the 3 and 10 day-old cultures of intervertebral discs (Fig. 2A and B) demonstrated that Col II expression in the group treated with inflammatory cytokines was markedly reduced compared with the blank control group. However, its expression was markedly increased by treatment with KGN.

In Fig. 2C and D, the paraffin sections of coronary regions of the mouse intervertebral discs were stained with $\mathrm{H} \& \mathrm{E}+$ Alcian blue. Aggrecan expression was inhibited by inflammatory cytokines (Fig. 2C and D) compared with the control group, but was obviously increased by adding KGN, particularly at the high concentration. In Fig. 3A-C, western blot analysis showed that the aggrecan expression in hNPCs and mouse intervertebral discs was increased by adding KGN, especially at a high concentration, and reduced by inflammatory cytokines.

The Col II expression in hNPCs and mouse intervertebral discs was further determined by western blot analysis. Results of the western blot analyses for the hNPCs and mouse intervertebral discs in Fig. 3A-C demonstrated that Col II expression in the group treated with inflammatory cytokines was significantly reduced compared with the control group. However, its expression was increased by varying degrees by the addition of KGN, particularly at the high concentration (Fig. 3A-C).

Effects of KGN on aggrecan and Col II gene expression. The $\mathrm{Col}$ II and aggrecan mRNA expression in hNPCs and mouse 

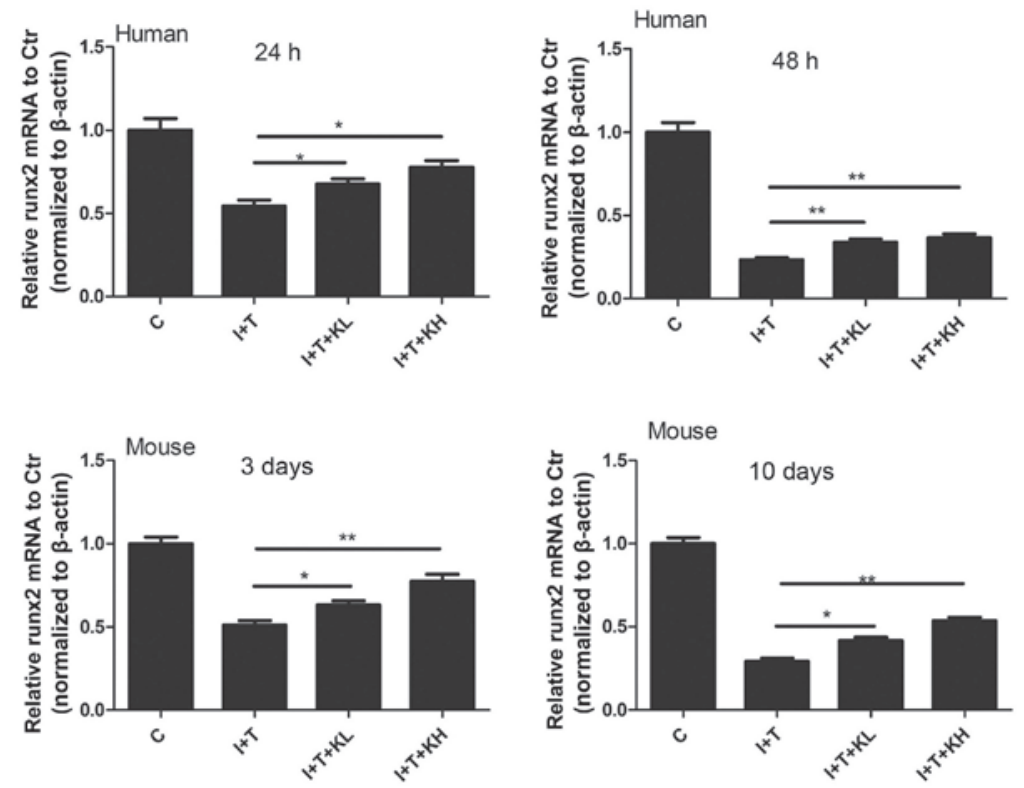

Figure 5. RUNX2 expression was elevated by KGN. RUNX2 expression in human nucleus pulposus cells and mouse intervertebral discs were inhibited by inflammatory cytokines. However, it was elevated after the treatment of KGN, especially at a high concentration ("P $<0.05$ and $\left.{ }^{* *} \mathrm{P}<0.001\right)$. RUNX2, runt related transcription factor 2 ; KGN, kartogenin; IL-1 $\beta$, interleukin- $1 \beta$; TNF- $\alpha$, tumor necrosis factor- $\alpha$; C, control; I+T, IL-1 $\beta+$ TNF- $\alpha$; I+T+KL, IL-1 $\beta+$ TNF- $\alpha+$ low concentration of KGN; I+T+KH, IL-1 $\beta+$ TNF- $\alpha+$ high concentration of KGN.

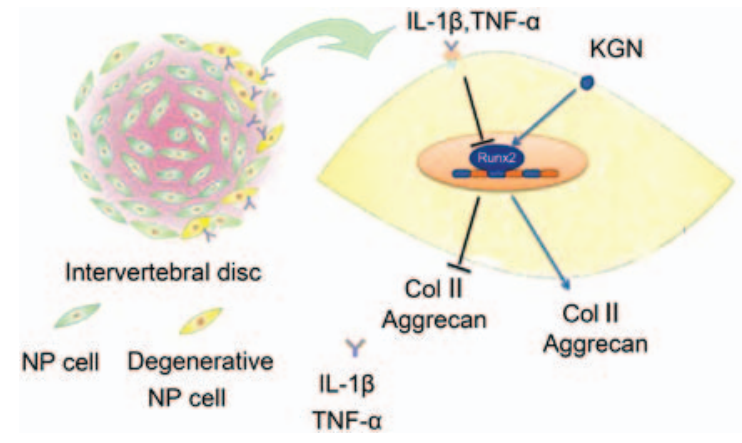

Figure 6. IL-1 $\beta$ and TNF- $\alpha$ improved the degeneration of NP cells and discs by interrupt the expression of RUNX2. KGN effectively promoted the expression of RUNX2, thus increased the Col II and aggrecan, and rivaled to the degeneration of disc induced by cytokines. KGN effectively delayed the degeneration of intervertebral disc. IL-1 $\beta$, interleukin-1 $\beta$; TNF- $\alpha$, tumor necrosis factor- $\alpha$; KGN, kartogenin; Col II, type II collagen; NP, nucleus pulposus; RUNX2, runt related transcription factor 2 .

NPCs was determined to identify whether KGN can increase the gene expression of $\mathrm{Col}$ II and aggrecan (Fig. 4A-H). In Fig. 4, the mRNA expression of $\mathrm{Col}$ II and aggrecan was reduced in the groups treated with inflammatory cytokines compared with the control group. However, their expression was increased following treatment with KGN, particularly at the high concentration.

Effects of KGN on the gene expression of RUNX2. To investigate the mechanism of KGN in secretion of Col II and aggrecan expression, RUNX2 mRNA expression in NPCs was determined. The expression of RUNX2 mRNA was reduced by the cytokines; whereas, it was significantly increased by co-treatment with the KGN and the cytokines, compared with cytokine treatment alone (Fig. 5).

IL- $1 \beta$ and TNF- $\alpha$ inhibited the secretion of Col II and aggrecan in NPCs and suppressed RUNX2 expression, which is the critical transcription factor required for Col II and aggrecan gene expression. KGN effectively increased the expression of RUNX2 and reversed the degeneration of NPCs induced by IL- $1 \beta$ and TNF- $\alpha$ (Fig. 6).

\section{Discussion}

The current study aimed to determine whether KGN can slow the degeneration of intervertebral discs. In degenerative intervertebral discs, the expression of several other members of the IL family is elevated, including IL-1, IL-2, IL-6, IL-8, IL-12 and IL-17. In addition, upregulated expression of interferon $\gamma$, nitric oxide (NO) and prostaglandin $\mathrm{E}_{2}\left(\mathrm{PGE}_{2}\right)$ released TNF- $\alpha$, inducible $\mathrm{NO}$, and PG, respectively, as detected in degenerative intervertebral discs. The expression of IL- $1 \beta$ and TNF- $\alpha$ is considered particularly important in intervertebral disc degeneration (19). IL-1 $\beta$ promotes the release of matrix metalloproteinase, which can degrade Col II and aggrecan (20), and affect the metabolism of cartilage matrix (21). TNF- $\alpha$ is associated with NPC apoptosis, disc protrusion and nerve root irritation (22).

Models have been established for the degeneration in intervertebral discs, such as the disc puncture model and the annulus fibrosus cutting model for animals standing upright. However, these models do not fully simulate the degeneration of intervertebral discs (23). In this study, a novel model for the degeneration in intervertebral discs was adopted. The in vitro organ culture of intervertebral discs effectively simulated the slow degeneration of intervertebral discs; a single intervertebral disc with the upper and lower endplates was dissected from mice and maintained in a culture medium containing IL-1 $\beta$ and TNF- $\alpha$ (24).

Johnson et al (16) used high-throughput screening and identified that KGN has a low molecular weight $(<1,000)$, as well as a simple structure, specific targets, and fewer side effects, thereby making it superior to macromolecular polypeptide drugs. KGN binds to fibroin A, thus disrupting it's binding to $\mathrm{CBF} \beta$. Genes 
of the Runt domain protein family are collectively referred to as 'RUNXX'. The transcription factor of the RUNXX family can bind to $\mathrm{CBF} \beta$, thus producing heterodimers and conferring a stronger ability to bind to DNA. CBF $\beta$ can bind to RUNX1, 2 and 3 and activate the transcription of Col II and aggrecan $(15,17)$. Takeda et al (25) reported that RUNX2 may promote the maturation of chondrocytes. The expression of RUNX2 is initially high in chondrocytes and the hypertrophic zone, thus inducing the hypertrophy of chondrocytes (26). Therefore, the expression of Col II and aggrecan are sustained in hypertrophic chondrocytes. Additionally, KGN may also induce the Col II secretion in dermal fibroblasts via the Smad4/5 pathway (27).

Notably, KGN may facilitate MSC differentiation into chondrocytes, maintain chondrocyte functions and promote the secretion of chondrocyte-associated proteins. Kang et al (17) recently reported that injection of KGN into the articular cavity regulates the regeneration of chondrocytes and promotes the expression of Col II and aggrecan. Xu et al (28) reported that KGN may effectively repair articular cartilage damage and elevate the expression of Col II and aggrecan. NPCs are cartilage-like cells with features similar to those of chondrocytes. Therefore, KGN may slow the degeneration of these cells.

Previous studies suggested that the degeneration of NPCs is a major cause of intervertebral disc degeneration. The increase or reduction of Col II and aggrecan secreted by NPCs may lead to the degeneration of intervertebral discs. Degeneration models using hNPCs and mouse intervertebral discs exposed to inflammatory cytokines have been established in the current study. The expression of Col II and aggrecan were evaluated using immunofluorescence, immunohistochemistry and western blot analysis. Particularly at high concentration used, KGN increased the expression of $\mathrm{Col}$ II and aggrecan to varying degrees. The mRNA expression of Col II and aggrecan was also altered by the inflammatory cytokines.

In addition, KGN treatment increased RUNX2 mRNA levels, which is the key transcription factor required for Col II and aggrecan expression in NPCs $(15,17)$.

The intervertebral disc is a specialized tissue that is relatively closed in vivo; all substances are slowly transported via small blood vessels in the two endplates and annulus fibrosus. However, the transport of drugs to the intervertebral discs following injection via the abdominal cavity of mice is extremely difficult (29). None of the in vivo mouse models of intervertebral disc degeneration can effectively simulate the slow degeneration of normal intervertebral discs. Thus, a limitation of the present study is that the effects of KGN on the degeneration of intervertebral discs under inflammatory cytokines may not be possible by direct injection in vivo. In addition, the ex vivo model used in the present study did not involve the two endplates, although KGN has the ability to regulate the two endplates. Unfortunately, the intervertebral disc has a special form. Specific in vivo biological reactions occur between the endplates and nucleus pulposus, and between the annulus fibrosus and nucleus pulposus. Studies confined to chondrocytes or NPCs cannot effectively simulate the presence of a nucleus pulposus with high permeability, low $\mathrm{pH}$ and hypoxemia in vivo. The present study used ex vivo organ cultures, where the end plates are retained following removal of the upper and lower vertebrae, thus effectively simulating the in vivo state of NPCs and the slow degeneration of intervertebral discs.
In conclusion, combined with previous studies on the effects of KGN on hNPCs, a potential method has been developed to slow the degeneration of intervertebral discs. However, a safe or effective drug has not yet been identified to eliminate intervertebral disc degeneration. To the best of our knowledge, the present study is the first to report that KGN can effectively slow the degeneration of intervertebral discs exposed to inflammatory cytokines. Therefore, in addition to being a safe and effective drug for treating osteoarthritis, KGN presents a novel approach to eliminate degeneration in intervertebral discs and produces new possibilities for investigating intervertebral disc degeneration under the actions of inflammatory cytokines.

\section{Acknowledgements}

This study was supported by grants from the NSFC (no. 81520108018).

\section{References}

1. Andersson GB: Epidemiological features of chronic low-back pain. Lancet 354: 581-585, 1999.

2. Katz JN: Lumbar disc disorders and low-back pain: socioeconomic factors and consequences. J Bone Joint Surg Am 88 (Suppl 2): 21-24, 2006.

3. Jenkins H: Classification of low back pain. Australas Chiropr Osteopathy 10: 91-97, 2002.

4. Le Maitre CL, Freemont AJ and Hoyland JA: The role of interleukin-1 in the pathogenesis of human intervertebral disc degeneration. Arthritis Res Ther 7: R732-R745, 2005.

5. Séguin CA, Pilliar RM, Roughley PJ and Kandel RA: Tumor necrosis factor-alpha modulates matrix production and catabolism in nucleus pulposus tissue. Spine 30: 1940-1948, 2005.

6. Le Maitre CL, Hoyland JA and Freemont AJ: Catabolic cytokine expression in degenerate and herniated human intervertebral discs: IL-1beta and TNFalpha expression profile. Arthritis Res Ther 9: R77, 2007.

7. Tian Y, Yuan W, Fujita N, Wang J, Wang H, Shapiro IM and Risbud MV: Inflammatory cytokines associated with degenerative disc disease control aggrecanase-1 (ADAMTS-4) expression in nucleus pulposus cells through MAPK and NF- $\mathrm{kB}$. Am J Pathol 182: 2310-2321, 2013.

8. Wang H, Tian Y, Wang J, Phillips KL, Binch AL, Dunn S, Cross A, Chiverton N, Zheng Z, Shapiro IM, et al: Inflammatory cytokines induce NOTCH signaling in nucleus pulposus cells: implications in intervertebral disc degeneration. J Biol Chem 288: 16761-16774, 2013.

9. Liu H, Pan H, Yang H, Wang J, Zhang K, Li X, Wang H, Ding W, Li B and Zheng Z: LIM mineralization protein-1 suppresses TNF-alpha induced intervertebral disc degeneration by maintaining nucleus pulposus extracellular matrix production and inhibiting matrix metalloproteinases expression. J Orthop Res 33: 294-303, 2015 .

10. Yang $X$ and Li X: Nucleus pulposus tissue engineering: a brief review. Eur Spine J 18: 1564-1572, 2009.

11. Wang SZ, Rui YF, Lu J and Wang C: Cell and molecular biology of intervertebral disc degeneration: current understanding and implications for potential therapeutic strategies. Cell Prolif 47: 381-390, 2014.

12. Vasiliadis ES, Pneumaticos SG, Evangelopoulos DS and Papavassiliou AG: Biologic treatment of mild and moderate intervertebral disc degeneration. Mol Med 20: 400-409, 2014.

13. Sakai D and Grad S: Advancing the cellular and molecular therapy for intervertebral disc disease. Adv Drug Deliv Rev 84: 159-171, 2015.

14. Masuda K: Biological repair of the degenerated intervertebral disc by the injection of growth factors. Eur Spine J 17 (Suppl 4): 441-451, 2008.

15. Marini JC and Forlino A: Replenishing cartilage from endogenous stem cells. N Engl J Med 366: 2522-2524, 2012.

16. Johnson K, Zhu S, Tremblay MS, Payette JN, Wang J, Bouchez LC, Meeusen S, Althage A, Cho CY, Wu X, et al: A stem cell-based approach to cartilage repair. Science 336: 717-721, 2012. 
17. Kang ML, Ko JY, Kim JE and Im GI: Intra-articular delivery of kartogenin-conjugated chitosan nano/microparticles for cartilage regeneration. Biomaterials 35: 9984-9994, 2014.

18. Livak KJ and Schmittgen TD: Analysis of relative gene expression data using real-time quantitative PCR and the 2(-Delta Delta C(T)) Method. Methods 25: 402-408, 2001.

19. Johnson ZI, Schoepflin ZR, Choi H, Shapiro IM and Risbud MV: Disc in flames: roles of TNF- $\alpha$ and IL-1 $\beta$ in intervertebral disc degeneration. Eur Cell Mater 30: 104-116, 2015.

20. Patel KP, Sandy JD, Akeda K, Miyamoto K, Chujo T, An HS and Masuda K: Aggrecanases and aggrecanase-generated fragments in the human intervertebral disc at early and advanced stages of disc degeneration. Spine 32: 2596-2603, 2007.

21. Videman T, Saarela J, Kaprio J, Näkki A, Levälahti E, Gill K, Peltonen L and Battié MC: Associations of 25 structural, degradative, and inflammatory candidate genes with lumbar disc desiccation, bulging, and height narrowing. Arthritis Rheum 60: 470-481, 2009

22. Igarashi T, Kikuchi S, Shubayev V and Myers RR: 2000 Volvo Award winner in basic science studies: exogenous tumor necrosis factor-alpha mimics nucleus pulposus-induced neuropathology. Molecular, histologic, and behavioral comparisons in rats. Spine 25: 2975-2980, 2000

23. Beierfuss A, Dietrich H, Kremser C, Hunjadi M, Ritsch A, Rulicke T, Thome C and Mern DS: Knockout of Apolipoprotein E in rabbit promotes premature intervertebral disc degeneration: A new in vivo model for therapeutic approaches of spinal disc disorders. PloS One 12: e0187564, 2017.

24. Pelle DW, Peacock JD, Schmidt CL, Kampfschulte K, Scholten DJ II, Russo SS, Easton KJ and Steensma MR: Genetic and functional studies of the intervertebral disc: a novel murine intervertebral disc model. PLoS One 9: e112454, 2014.
25. Takeda S, Bonnamy JP, Owen MJ, Ducy P and Karsenty G: Continuous expression of $\mathrm{Cbfal}$ in nonhypertrophic chondrocytes uncovers its ability to induce hypertrophic chondrocyte differentiation and partially rescues Cbfal-deficient mice. Genes Dev 15: 467-481, 2001.

26. Studer D, Millan C, Öztürk E, Maniura-Weber K and Zenobi-Wong M: Molecular and biophysical mechanisms regulating hypertrophic differentiation in chondrocytes and mesenchymal stem cells. Eur Cell Mater 24: 118-135, 2012.

27. Wang J, Zhou J, Zhang N, Zhang X and Li Q: A heterocyclic molecule kartogenin induces collagen synthesis of human dermal fibroblasts by activating the smad4/smad5 pathway. Biochem Biophys Res Commun 450: 568-574, 2014.

28. Xu X, Shi D, Shen Y, Xu Z, Dai J, Chen D, Teng H and Jiang Q: Full-thickness cartilage defects are repaired via a microfracture technique and intraarticular injection of the small-molecule compound kartogenin. Arthritis Res Ther 17: 20, 2015.

29. Kepler CK, Ponnappan RK, Tannoury CA, Risbud MV and Anderson DG: The molecular basis of intervertebral disc degeneration. Spine J 13: 318-330, 2013.

This work is licensed under a Creative Commons Attribution-NonCommercial-NoDerivatives 4.0 International (CC BY-NC-ND 4.0) License. 